\title{
Belgeo
}

Revue belge de géographie and prospects

\section{Les chemins de fer en Afrique subsaharienne, entre passé révolu et recompositions incertaines}

Railroads in Subsaharan Africa, between past days and uncertain

reconstructions

Roland Pourtier

(2) OpenEdition

Journals

Édition électronique

URL : http://journals.openedition.org/belgeo/11266

DOI : 10.4000/belgeo.11266

ISSN : 2294-9135

Éditeur :

National Committee of Geography of Belgium, Société Royale Belge de Géographie

Édition imprimée

Date de publication : 30 juin 2007

Pagination : 189-202

ISSN : 1377-2368

Référence électronique

Roland Pourtier, "Les chemins de fer en Afrique subsaharienne, entre passé révolu et recompositions incertaines », Belgeo [En ligne], 2 | 2007, mis en ligne le 10 décembre 2013, consulté le 19 avril 2019. URL : http://journals.openedition.org/belgeo/11266; DOI : 10.4000/belgeo.11266

Ce document a été généré automatiquement le 19 avril 2019

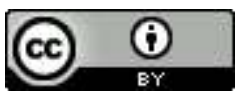

Belgeo est mis à disposition selon les termes de la licence Creative Commons Attribution 4.0 International. 


\title{
Les chemins de fer en Afrique subsaharienne, entre passé révolu et recompositions incertaines
}

\author{
Railroads in Subsaharan Africa, between past days and uncertain \\ reconstructions
}

Roland Pourtier

\section{Introduction}

1 Les chemins de fer africains ont connu leur heure de gloire dans la première moitié du XX ${ }^{e}$ siècle. Ils furent un des grands outils d'une mise en valeur coloniale, peu regardante sur le coût humain de chantiers ferroviaires ouverts dans des environnements éprouvants : la traversée du Mayombe, qu'il s'agisse du C.F.M.K. (Chemin de Fer Matadi-Kinshasa) ou du C.F.C.O. (Chemin de fer Congo-Océan) fut une rude épreuve (Sautter, 1967). L'épopée du rail laissa derrière elle de nombreuses victimes, mais quand le train eut vaincu les obstacles, quand les locomotives crachant leurs fumées se mirent à traverser forêts et savanes, les populations surent qu'un nouveau temps était arrivé. Ce temps est aujourd'hui révolu. Les vieux cheminots s'en souviennent encore avec nostalgie, ceux du Djibouti-Addis Abeba, ou ceux qui déploient encore des trésors d'ingéniosité pour maintenir en survie le peu qui reste de la Société nationale des chemins de fer congolais (SNCC). La roue a tourné en faveur de la route.

2 Le diagnostic général des chemins de fer africains ne prête pas à l'optimisme. Pour un réseau continental totalisant environ $90000 \mathrm{~km}$, plus de lignes ferment qu'il ne s'en crée de nouvelles. Quand il est encore possible, le voyage en train se révèle très aléatoire. Le luxueux Blue Train qui circule entre Pretoria et Le Cap et sur la Garden Route entre Port Elisabeth et Le Cap fait exception (Robbins, 1993). Ailleurs, vétusté du matériel roulant, vitesse commerciale réduite sur des voies mal entretenues, horaires non respectés etc. détournent les voyageurs du rail. Au Nigeria (Jaekel, 1997), pourtant le seul pays hors 
d'Afrique australe à disposer d'un véritable réseau (totalisant $3505 \mathrm{~km}$ de voies), le fret ferroviaire ne cesse de perdre des parts de marché au bénéfice du transport routier. Le trafic passager a considérablement diminué: en 2005, la compagnie nationale de transport ferroviaire, Nigeria Railway Corporation, n'assurait plus que quelques liaisons dont Lagos-Kano et Port-Harcourt Kano deux fois par semaine. Dans plusieurs pays, les guerres ont porté un coup très dur au trafic ferroviaire. En Angola comme au Mozambique les infrastructures ferroviaires en portent les séquelles. La partition de la Côte d'Ivoire depuis 2002 paralyse l'Abidjan-Niger, en réduisant à néant les efforts accomplis depuis la privatisation de 1995. Même des réalisations récentes comme le Tazara (Tanzania-Zambia Railway) ou le Transgabonais connaissent des difficultés. Le chemin de fer en Afrique serait-il condamné ? Y a-t-il encore un avenir pour un mode de transport affecté par la crise multiforme qui touche la plupart des Etats subsahariens à l'exception de l'Afrique du sud? Cette exception sud-africaine ne montre-t-elle pas, précisément, une corrélation forte entre l'état du transport ferroviaire et le niveau global de développement ? Autant de questions auxquelles on tentera d'apporter des éléments de réponse, forcément incomplets et partiels, car il ne faut pas oublier que l'Afrique est plurielle et qu'une réflexion qui se veut générale ne peut rendre compte de tous les cas particuliers $^{(1}$

\section{Le poids des héritages}

3 Comme pour toute question concernant l'Afrique Noire, l'évaluation des héritages coloniaux reste indispensable, en dépit de l'éloignement croissant de la période coloniale (Almeida-Topor, 1992). Cela est particulièrement vrai dans le domaine ferroviaire, car la plupart des infrastructures ont été mises en place avant le tournant des indépendances. Elles ont été pensées en fonction des configurations spatiales issues du partage de l'Afrique. Celui-ci est responsable de leur aspect éclaté, et d'un manque général de cohérence.

\section{Des embryons de réseaux}

4 Un simple regard sur une carte des tracés ferroviaires africains (figure 1) montre la quasiabsence de réseaux, l'Afrique du sud, avec un réseau de $30600 \mathrm{~km}$ de voies ferrées et les infrastructures ferroviaires les plus performantes d'Afrique subsaharienne devant être mise à part (South Africa Yearbook, 2003). Cet état de fait résulte des politiques d'équipement mises en oeuvre par les administrations coloniales, avec pour seul cadre de référence le territoire de chacune des colonies, au détriment d'une vision continentale ou tout au moins régionale. L'Afrique de l'ouest, la partie du continent où le partage colonial s'est traduit par le plus fort morcellement territorial, en est l'illustration, jusqu'à la caricature dans le cas d"'Etats-tranches" comme les actuels Bénin et Togo. Voisins et rivaux, les Français et les Allemands construisirent des pénétrantes ferroviaires parallèles à partir d'étroites ouvertures sur le Golfe de Guinée : à la ligne Cotonou-Parakou répond la ligne Lomé-Sokodé. 
Figure 1. Des réseaux ferroviaires fragmentés.

Des réseaux ferroviaires fragmentés

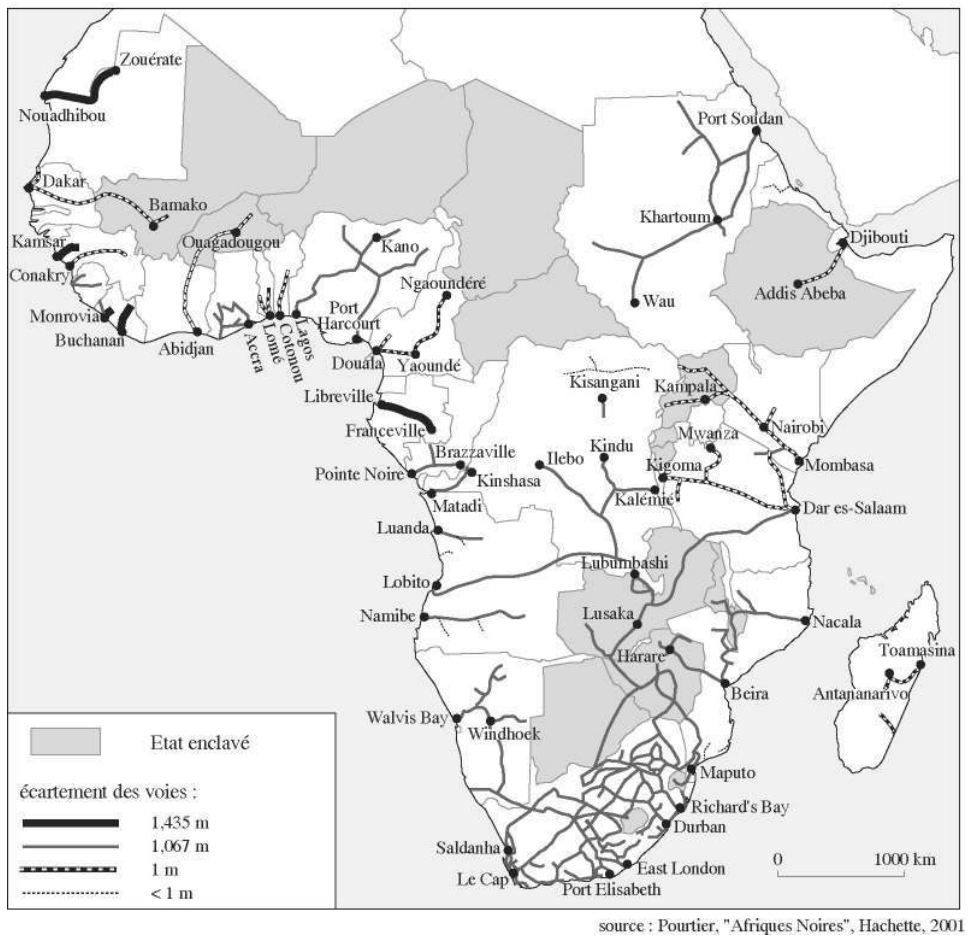

5 La faible structuration de l'espace associée à la quasi absence de maillage urbain (à de rares exceptions près comme les villes yoruba et haoussa au Nigeria) n'offrait pas de véritables lignes directrices susceptibles d'orienter le choix des infrastructures. La transposition des nationalismes européens sur le continent africain allait en outre fait obstacle à la recherche d'une "rationalité" continentale dans l'architecture ferroviaire. Les différences d'écartement des voies en sont l'éloquent témoignage. Les voies ferrées coloniales sont des voies métriques (sans compter les "decauville" de $60 \mathrm{~cm}$ d'écartement utilisés dans les mines et carrières et, au Congo, pour la desserte rurale de la région des Uele). Mais le mètre dont les Français pensaient depuis la révolution que l'étalon avait valeur universelle fut autrement interprété par les Britanniques qui imposèrent un écartement de $1,067 \mathrm{~m}$. dans leurs colonies africaines. Toute l'Afrique australe et jusqu'à l'Angola et au Congo belge se mit au diapason anglais. Les colonies allemandes suivirent le standard français. Il en est résulté d'impossibles interconnexions. La voie "métrique", en dépit de ses performances limitées, a été retenue pour la construction, postérieure aux indépendances, du "Tazara", construit par la Chine, qui ouvre la Zambie sur l'Océan Indien via le port de Dar es-Salaam. La majorité des infrastructures ferroviaires postérieures aux années 1950 est cependant à écartement dit "normal” (1,435 m) : ce standard des voies européennes a été adopté pour les convois minéraliers lourds. Les différents écartements ne sont pas faits pour faciliter l'établissement de réseaux à l'échelle régionale ou continentale. En réalité, seule l'Afrique du sud possède un véritable réseau, dense et opérationnel, qui se prolonge par des appendices pénétrant l'ensemble de l'Afrique australe, Zimbabwe, Zambie (et au-delà Katanga), Mozambique, Namibie. 


\section{Etats littoraux et états enclavés}

6 Outils de contrôle territorial et de mise en valeur, les chemins de fer sont montés à l'assaut du continent à partir des bases portuaires de la pénétration coloniale. Sur le plan spatial, cette double finalité, politique et économique, permettait de désenclaver les pays de l'intérieur de façon à les intégrer à l'économie-monde. Le chemin de fer a été le premier instrument d'intégration territoriale, le premier outil de ce qu'on n'appelait pas encore le développement. A la fin du XIX ${ }^{e}$ siècle, la voie ferrée et la machine à vapeur, fer de lance du progrès, étaient porteuses de rêves prométhéens. L'Afrique, considérée par nombre de théoriciens de la colonisation comme une "tabula rasa", représentait un terrain propice à l'application des principes d'action devant permettre l'exploitation des ressources naturelles au profit des métropoles en même temps que la diffusion de la "civilisation". L'idéologie du rail était proclamée sans ambages. On peut encore voir, à proximité de Matadi, un texte gravé dans la pierre, proclamant la gloire du chemin de fer qui "ouvrit le bassin du Congo à la civilisation". Non loin de là, un "Monument aux porteurs" commémore l'infrastructure qui "les libéra du portage".

7 L'enclavement est une question d'échelle et d'accessibilité. Ainsi le Katanga est enclavé à l'intérieur du territoire de la RDC, mais aussi par rapport à la configuration continentale. Les stratégies ferroviaires destinées à rendre possible l'exploitation des minerais du Copperbelt ont été pensées selon cette double référence, nationale et continentale. L'exploitation du cuivre a commencé en 1910 à Lubumbashi dès qu'un raccordement ferroviaire avec le réseau d'Afrique australe a permis le transport du cuivre vers les ports d'Afrique du sud (East London, Port Elisabeth). Toute la problématique de la "voie nationale", expression de ce qu'on peut appeler le nationalisme colonial, a résidé dans la volonté de construire un système de transport entre le Katanga et Matadi - et au-delà jusqu'à Anvers - entièrement en "territoire belge", avant que le Congo indépendant en assume l'héritage (Lederer, 1982, Charlier 1993). Il fallait réduire la dépendance vis-à-vis des pays de transit et d'exportation. Mais l'équipement en voies ferrées ne fut pas poussé jusqu'à son terme, l'option du transport bimodal fluvio-ferroviaire s'étant imposée : ainsi, d'Ilebo (ex-Port Franqui) à Kinshasa la voie fluviale Kasai-Congo s'interpose entre le B.C.K. et le C.F.M.K. Les partisans d'une liaison ferroviaire continue du Katanga à Matadi (Jadot, 1952) ne purent imposer leurs vues. Depuis l'indépendance de la RDC, ce projet, tel un serpent de mer, refait cependant périodiquement surface (Murairi J.B., 1970), mais ce n'est assurément pas un priorité pour la reconstruction du territoire congolais.

8 Les réalisations ferroviaires "régionales", selon l'acception qui s'est imposée pour désigner des regroupements entre plusieurs Etats, remontent au temps de la colonisation. Leur objectif était de désenclaver les colonies privées d'accès à la mer en demeurant à l'intérieur du cadre territorial et juridique des fédérations. En Afrique occidentale française (AOF), le "Dakar-Niger" qui relie Dakar à Bamako a été conçu pour ouvrir le vaste Soudan (actuel Mali) à l'économie de marché (Lakroum, 1987). Dans la même logique, l'Abidjan-Niger a créé une relation forte entre Abidjan et Ouagadougou via Bobo Dioulassso et préfiguré le rattachement à la Côte d'Ivoire, sous le nom de "Haute Côte" de la plus grande partie de la Haute-Volta lorsque celle-ci fut démantelée en $1937^{2}$. En Afrique équatoriale française (AEF) en revanche aucun des grands projets régionaux n'a abouti. La construction d'une liaison ferroviaire Libreville-Ouesso avait été décidée par le gouvernement français en 1913, avec pour perspective à plus long terme d'atteindre 
Bangui. La Guerre de 1914-1918 mit un terme définitif au projet et c'est l'axe BrazzavillePointe-Noire qui fut privilégié après la guerre : le "Congo-Océan", (le chantier devait durer de 1921 à 1934) double, sur la rive droite du fleuve Congo, le chemin de fer belge Kinshasa-Matadi. Après 1945, un nouveau projet ferroviaire, le "Bangui-Tchad" fut mis à l'étude. Il devait compléter jusqu'à Fort-Lamy (N’Djamena) la "voie transéquatoriale" composée du Congo-Océan et de l'axe navigable Brazzaville-Bangui. Les aspects socioéconomiques de ce projet furent étudiés par Gilles Sautter. Il en est resté une belle étude de géographie tournée vers l'action, mais le projet ferroviaire resta dans les cartons (Sautter, 1958).

9 En Afrique orientale, les Britanniques eurent aussi leur grand projet régional avec la liaison Dar es-Salaam- Kampala. L'objectif déclaré était de désenclaver le royaume de Buganda, considéré comme la perle de l'empire dans l'est de l'Afrique (Miller, 1971). A miparcours, une base technique créée en 1902 est à l'origine de Nairobi : exemple le plus remarquable, parmi d'autres, des effets induits du chemin de fer, créateur de villes, ici selon une logique assez particulière découlant des contraintes liées à la construction de la voie ferrée. Un autre projet de désenclavement, contemporain de celui-ci, est le chemin de fer Djibouti-Addis Abeba (1897-1917) construit par les Français (Gascon, 2006). Les mêmes raisons techniques ont donné naissance à une ville de mi-parcours, Dirré Dawa, ville des cheminots, qui sans avoir l'importance de la capitale millionnaire du Kenya compte tout de même aujourd'hui plus de 200000 habitants.

10 Les chemins de fer coloniaux ont joué un rôle important dans l'urbanisation de l'Afrique Noire. Il s'agit tantôt de villes port, comme Abidjan qui s'imposa comme capitale en devenant tête de ligne ferroviaire, ou encore Cotonou, Mombasa ou Dar es-Salaam; tantôt de relais techniques et, dans les cas de loin les plus nombreux, de marchés créés autour des gares. Incontestablement, les chemins de fer ont été un remarquable instrument de structuration des territoires, mais, sauf en Afrique du sud, ils n'ont pas atteint le stade d'un véritable aménagement réticulaire: le linéaire l'emporte sur la composition en réseau.

\section{Le rail, la mine et le territoire}

11 Parmi les principales fonctions dévolues au transport ferroviaire en Afrique, les unes relèvent de l'aménagement du territoire, dans une acception globale, les autres font de la voie ferrée un simple prolongement de l'exploitation minière.

\section{Le chemin de fer exutoire de pondéreux}

12 L'un des trains les plus longs du monde, jusqu'à $2,5 \mathrm{~km}$, transporte le minerai de fer mauritanien de Zouérate à Nouadhibou. Exemple parfait d'une infrastructure créée de toutes pièces articulant mine, voie ferrée, port. Mis en exploitation en 1963 par la MIFERMA (Société anonyme des mines de fer de Mauritanie) le complexe minier est géré, depuis sa nationalisation, par la SNIM (Société nationale industrielle et minière) (Bonte, 2001). Traversant le désert, le chemin de fer n'a qu'un rôle insignifiant de desserte régionale. Le jour où la mine fermera, marquera aussi son arrêt, le wagon touristique accroché aux convois minéraliers ne justifiant évidemment pas le maintien de l'infrastructure. Les mêmes constats peuvent être faits partout où la voie ferrée n'est rien d'autre qu'un exutoire de la mine, par exemple en Guinée pour le transport de la bauxite 
de Sangarédi vers le port de Kamsar et de Fria vers celui de Conakry. En Afrique du sud, la Coal Line transporte le charbon des mines du Mpumalanga vers le port spécialisé de Richard's Bay (le volume transporté a atteint le milliard de tonnes en décembre 2000) ; le fer des mines de Sishen est acheminé par voie ferrée vers Saldanha Bay. Les voies ferrées minéralières peuvent aussi être utilisées pour le transport de marchandises générales et de voyageurs: ces infrastructures à vocations multiples participent des politiques d'aménagement du territoire.

\section{Les corridors d'intégration régionale et de développement}

13 En Afrique australe et orientale, les politiques de développement régional recherchent la meilleure intégration possible entre transport et création d'activités économiques, dans les domaines agricoles et industriels. Le modèle des corridors de développement s'appuie sur des transports multimodaux, route et voie ferrée devant remplir des fonctions complémentaires, notamment dans la répartition du fret, la lenteur du transport ferroviaire étant compensée par un coût plus faible que celui du transport routier. En Afrique orientale, le transport ferroviaire représente un quart du fret marchandise ; il a trouvé son créneau dans le transport de pondéreux et de produits non périssables. Les corridors sont aussi des outils d'intégration régionale et de désenclavement. Par exemple, l'accès aux Pays des Grands Lacs s'effectue par deux corridors. Dans le corridor nord, via le Kenya, route et rail suivent le même tracé, de Mombasa à Kampala. Dans le corridor sud qui traverse la Tanzanie, les itinéraires ferroviaires et routiers, moins proches les uns des autres, relient Dar es-Salaam au lac Tanganyika, donnant accès au Burundi et au Sud Kivu.

14 En Afrique australe, la reconstruction économique du Mozambique et son intégration régionale s'appuient sur trois corridors. Le corridor nord relie par voie ferrée le Malawi au port de Nacala (Béranger, 2004). Le corridor de Beira, comprenant route, voie ferrée et oléoduc, est la principale ouverture océanique du Zimbabwe. Le corridor de Maputo sert de débouché à la fois pour le Zimbabwe (par une voie ferrée longeant la vallée du Limpopo) et pour la région du Gauteng en Afrique du sud. Le transport ferroviaire est ainsi partie prenante d'un développement intégré. La politique des corridors fondée sur la complémentarité entre transport routier et transport ferroviaire participe d'une approche pragmatique et bénéficie de l'expérience en matière de gestion des réseaux ferroviaires de l'Afrique du sud.

\section{Le Transgabonais, entre mine et territoire}

Le chemin de fer Transgabonais est une des rares infrastructures ferroviaires récentes : achevé en 1987, il relie Libreville à Franceville, traversant en diagonale le territoire gabonais dont il constitue un axe structurant majeur (figure 2). Il s'agit d'un chemin de fer à vocation minéralière, ce qui explique l'écartement des voies au standard de 1,435 m. Le projet initial, étudié à la fin des années 1960, était lié à l'exploitation des mines de fer de Belinga, à l'extrême nord-est du pays. On est donc en présence du schéma classique de l'économie extractive d'exportation fondée sur le système mine-rail-port. Au cours des années 1950 un projet symétrique avait vu le jour: l'exploitation du manganèse de Moanda, dans le Haut-Ogooué, par la Compagnie minière de l'Ogooué (COMILOG). La Banque mondiale avait accordé un prêt pour la construction d'un système de transport, opérationnel à partir de 1962, comprenant un téléphérique entre la mine et la frontière 
congolaise, une voie ferrée de raccordement au Congo-Océan et le port minéralier à Pointe-Noire. Sur le même modèle, la Société des mines de fer de Mekambo (SOMIFER) sollicita la Banque mondiale pour le financement d'un "Transgabonais" qui devait relier la mine de Belinga à un port minéralier à construire à la pointe de Santa Clara, au nord de Libreville. Or en 1972, la Banque mondiale refusa de financer une infrastructure que la concurrence sur le marché international du fer, notamment celle du Brésil, ne justifiait pas.

Figure 2. Le Transgabonais : entre mine et territoire.

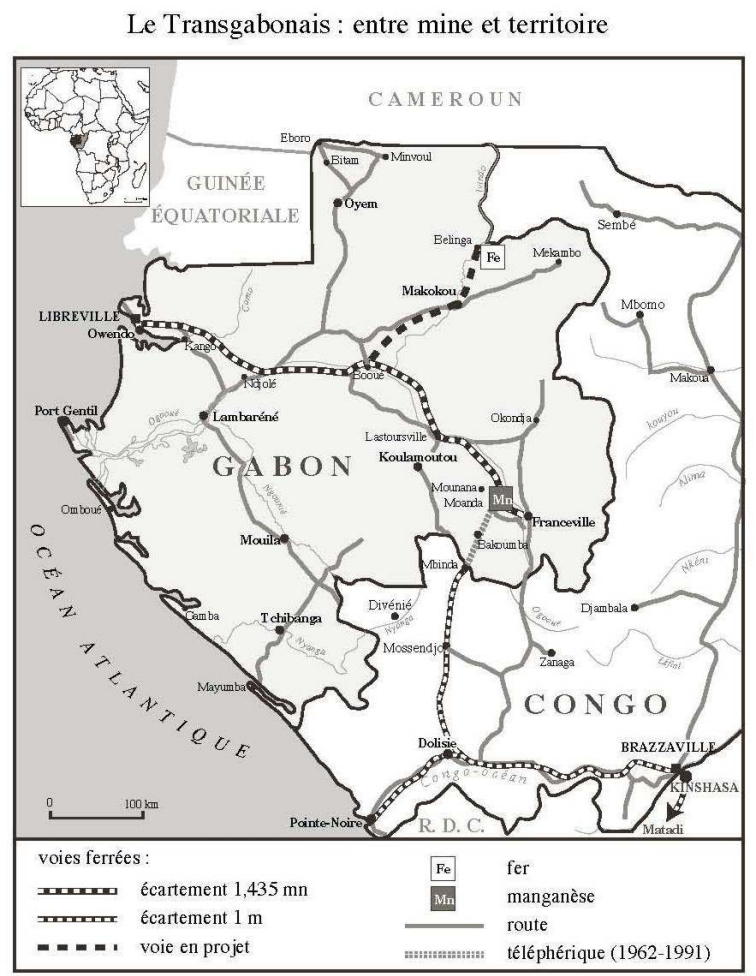

Le Transgabonais devint alors un problème politique à forte symbolique territoriale (Pourtier, 1982). Priver le Gabon d'un chemin de fer, alors que le Cameroun terminait le prolongement du Transcamerounais de Yaoundé à Ngaoundéré, fut ressenti comme une atteinte au prestige national. Un discours idéologique se greffa sur le projet, le chemin de fer étant présenté comme "le ciment de l'unité nationale", et le vecteur du développement de l'intérieur du pays, avec notamment les perspectives d'exploitation des forêts encore intactes car trop éloignées du littoral. Le projet fut sauvé par le pétrole et les recettes inattendues consécutives au "choc pétrolier" de 1973-1974: le quadruplement des cours du brut se traduisit par un triplement des recettes de l'Etat qui se trouva en mesure de se passer de la Banque mondiale. De fait, c'est le Gabon qui finança les travaux de génie civile, des prêts de pays européens et de divers organismes bancaires apportant les financements complémentaires. Toutefois, le marché du fer demeurant déprimé, le tracé du Transgabonais fut réorienté, sous l'impulsion du Président de la République gabonaise Omar Bongo en direction du Haut-Ogooué : en 1987, la liaison Libreville-Franceville était achevée.

17 Ce nouveau tracé du Transgabonais répond à des considérations géopolitiques bien plus qu'économiques. En établissant un lien fort entre la capitale, Libreville, et la province 
périphérique du Haut-Ogooué (une province qui avait été rattachée à la colonie du Moyen-Congo de 1925 à 1946) il participe à la construction nationale. Il est aussi l'oeuvre du Prince: le Président Bongo natif d'un village proche de Franceville manifeste sa réussite en dotant la terre de ses ancêtres du prestigieux chemin de fer qui fut alors chanté et dansé, fit l'objet de toutes sortes de dithyrambes. Enfin, le Transgabonais allait permettre de transporter le minerai de manganèse de Moanda au port minéralier d'Owendo en mettant un terme à la dépendance que représentait le transit par le Congo et Pointe-Noire. D'une certaine façon, le chemin de fer a achevé la construction du territoire national gabonais inscrite dans la disparition de la fédération d'AEF au tournant des indépendances. En 1991, un accident ferroviaire sur le CFCO fut le prétexte pour l'abandon définitif de la "voie congolaise", un abandon en tout état de cause programmé par la COMILOG

18 Avec un fret marchandise compris entre 3 et 3,5 millions de tonnes par an (dont manganèse 2 millions de $\mathrm{T}$., bois 1 million de T.) et quelque 300000 voyageurs transportés, le Transgabonais est loin d'assurer sa rentabilité. Le fonctionnement est obéré par des coûts de gestion insupportables qui ont conduit à la privatisation. L'avenir se présente cependant sous de meilleurs auspices: le renversement de tendance du marché des matières premières, suite notamment à la forte demande de la Chine, revalorise le gisement de Belinga qui n'attend plus pour être exploité que la construction du tronçon ferroviaire Booué-Belinga dont le marché a été attribué à la China National Machinery and Equipement Import and Export Ccorporation (C.M.E.C.). Le nouveau contexte minier va permettre la réalisation du projet initial de Transgabonais, après quarante ans d'attente. Les tribulations du chemin de fer soulignent la dépendance du Gabon - la même chose valant pour tous les pays africains hors Afrique du sud - envers les centres dominants de l'économie mondiale, les pays du Nord désormais rejoints par les pays émergents.

\section{Débâcle ferroviaire et désagrégation de l'Etat en RDC}

19 Les chemins de fer de la RDC ont pratiquement cessé de rouler. Seul le Chemin de fer Matadi-Kinshasa participe encore, tant bien que mal, aux activités de transport entre la capitale congolaise et son débouché maritime. Les temps ont bien changé depuis la déclaration fameuse de Stanley: "sans chemins de fer le Congo ne vaut pas un penny". Dès 1898 la voie ferré contournant les rapides du cours inférieur du fleuve remplissait son rôle d'artère vitale en formant le premier maillon d'un système de transport ferro-fluvial unique en Afrique (Huybrechts, 1970). Aujourd'hui, la clé de voûte du système de transport congolais n'a plus qu'une fonction marginale (Charlier et M'pene, 2006). Le trafic marchandises, autour de 200000 tonnes au début des années 2000, représente moins du dixième de ce qu'il était à la veille de l'indépendance. L'approvisionnement de Kinshasa et de son arrière-pays en produits importés dépend essentiellement de la route : l'arrêt du transport ferroviaire n'entraînerait pas de graves perturbations pour la vie quotidienne des millions d'habitants de la capitale congolaise.

Le CFMK ne remplit plus sa fonction d'exportation des minerais du Katanga depuis le quasi-arrêt de la production cuprifère de la GECAMINES au cours des années 1990, et la paralysie simultanée du réseau de la Société nationale des chemins de fer zaïrois (SNCZ). Le système de transport ferro-fluvial constitutif de la "voie nationale" s'est effondré (Pourtier, 1993). Si une reprise de l'activité extractive est en cours, l'exportation ne 
s'effectue pas par le territoire congolais mais par le réseau de transport routier et ferroviaire de l'Afrique australe. Le cobalt prend le chemin de Durban. La relance de l'exploitation du cuivre dépend largement de la réhabilitation du chemin de fer de Benguela, voie la plus courte et la plus économique entre le Katanga et le port atlantique de Lobito. La complémentarité entre transport minéralier et desserte du territoire congolais a vécu. Quelques rares trains circulent encore, de Lubumbashi vers Kananga ou vers Kindu et Kalémié. Prouesses de cheminots, qui maintiennent le rail en survie parce que le réseau routier très dégradé ne vaut guère mieux. La reconstruction du Congo, après une décennie de troubles et de guerres qui ont porté le coup de grâce à l'Etat moribond légué par Mobutu, devrait privilégier le fleuve et la route : l'aide internationale va dans ce sens. L'avantage du rail n'est avéré que pour le transport des pondéreux. Seul le Katanga est concerné : étant donné sa situation géographique, c'est dans le cadre régional de l'Afrique australe élargie à l'Angola et de l'Afrique orientale qu'une reprise du transport ferroviaire peut être envisagée.

21 Il est peu probable en effet que le modèle fluvio-ferroviaire d'origine coloniale puisse renaître, et irréaliste de penser qu'un prolongement de la voie ferrée d'Ilebo à Kinshasa pourrait résoudre les problèmes de transport du Katanga dans le cadre territorial de la RDC. Les différents programmes de réhabilitation des chemins de fer zaïrois engagés sous l'égide de la Banque mondiale dans les années 1970 et 1980 n'ont pas donné les résultats escomptés. Les réformes de la SNCZ et de l'ONATRA (Office national des transports qui gère le CFMK) n'ont pas été concluantes. Fragilisés pour des raisons structurelles (vétusté des matériels, manque d'entretien des voies, mauvaise gestion, pléthore de personnel), les transports ferroviaires se sont effondrés au cours des années 1990, cette décennie du chaos qui a achevé de déstructurer le territoire congolais happé dans une spirale de sousdéveloppement. La reconstruction de l'Etat, amorcée par la normalisation politique de 2006, au terme d'une "transition" douloureuse, impose celle des infrastructures de communication, condition sine qua non de l'effectivité du contrôle territorial et de la relance de l'économie d'échange (M'Pene, 2002). Dans ce vaste chantier, la priorité va à la route plus qu'à des chemins de fer d'un autre âge, même si le "Programme multisectoriel de reconstruction" lancé en 2003 avec l'appui de la Banque mondiale fait mention de projets de modernisation et d'extension du réseau ferré: on mesure là le poids des schémas hérités. Plus réaliste apparait la recommandation de l'Union africaine des Chemins de fer lors de sa $30^{\mathrm{e}}$ assemblée générale tenue à Kinshasa en 2003 de renforcer les liaisons inter-Etats, en réhabilitant notamment le chemin de fer de Benguela: la logique régionale et minière marque des points.

\section{Quel avenir pour le rail africain?}

22 Le Congo représente un cas extrême, le panorama ferroviaire n'est pas partout aussi sombre. Toutefois, les avantages comparatifs du chemin de fer apparaissent partout de plus en plus limités et tendent à se restreindre à la sphère du transport des pondéreux.

\section{L'irrésistible concurrence de la route}

Le transport de voyageurs se détourne chaque année un peu plus du chemin de fer. En réalité, celui-ci ne joue encore un rôle notable que dans la desserte d'agglomérations urbaines, par exemple en Afrique du sud. Les liaisons inter-urbaines lui échappent au 
rythme de l'aménagement des réseaux routiers. C'est le cas depuis déjà plus d'une décennie au Nigeria. Dans tous les pays disposant d'un réseau routier performant l'évolution va dans le même sens. En Afrique australe, malgré la relative densité du réseau ferroviaire, les déplacements de personnes s'effectuent essentiellement par route. Partout les services d'autocars relayent le train, l'animation des gares routières tranche avec l'atonie, sinon la fermeture, des gares ferroviaires. L'avantage de la route est évident, diversité des itinéraires, rapidité, confort, régularité horaire sur les grands axes. Différentes catégories de véhicules, du mini-bus aux cars climatisés offrent des prestations pour toutes les bourses. Les "taxis-brousse" surchargés, les camions brinquebalants chargés de grappes humaines circulent toujours certes sur les pistes rurales où tout déplacement tient encore de l'aventure, mais le renforcement constant du réseau de routes asphaltées contribue fortement à modifier l'économie du transport et les pratiques des usagers. L'achèvement en cours du bitumage sur l'axe Dakar-Bamako, réduit le transport voyageur, déjà pratiquement abandonné au Sénégal, à peu de chose et contraint à réévaluer l'utilité du chemin de fer. Autre exemple : sur la liaison YaoundéDouala, une société de transport propose des départs toutes les heures de $5 \mathrm{~h}$ à $19 \mathrm{~h}$, en cars climatisés munis de ceintures de sécurité pour chaque passager. A ce transport haut de gamme s'ajoute l'offre diversifiée de multiples compagnies. Avec ses deux liaisons quotidiennes aux horaires incertains, le train ne fait plus recette. Il n'a résisté à la concurrence qu'aussi longtemps que les pouvoirs publics ont bloqué le bitumage de la route dans un combat d'arrière-garde. Le Transcamerounais conserve en revanche tout son intérêt comme voie de désenclavement du Tchad et de la RCA: à partir de Ngaoundéré, la route prend le relais vers N'Djamena et Bangui, la conteneurisation facilitant le transport multimodal le long de la chaîne port-rail-route.

\section{Déficits de gestion et privatisations}

Les défaillances gestionnaires sont souvent présentées comme responsables, au moins partiellement, des difficultés que rencontre le transport ferroviaire. L'exception, encore une fois, est l'Afrique du sud et sa société publique Transnet dont le département Spoornet gère le réseau ferré. Dans la plupart des pays africains les infrastructures ferroviaires sont la propriété de l'Etat et la gestion du transport relève de sociétés nationales. Toutes les études, qu'elles émanent de la Banque mondiale ou de bureaux de consultants, insistent sur le peu d'efficacité et le coût disproportionné d'une gestion qui souffre des maux habituels des sociétés publiques en Afrique, détournements de fonds, emplois pléthoriques, absence de vision à long terme. Le chemin de fer ne peut s'accommoder d'une gestion au jour le jour. Or, penser le futur, un des fondements du capitalisme, n'est pas toujours entré dans les pratiques africaines. C'est pourquoi, pour remédier aux dysfonctionnements généralisés des chemins de fer, la privatisation accompagnée d'une gestion souvent confiée à des groupes non-africains (par exemple Bolloré) est apparue, dans la foulée des politiques d'ajustement structurel, comme l'ultime recours pour sauver le transport ferroviaire. Il n'existe toutefois ni solution miracle, ni modèle unique de privatisation. En Côte-d'Ivoire, les difficultés de l'ancienne Régie des chemins de fer Abidjan-Niger (RAN) ont conduit en 1989 à son éclatement en deux sociétés, l'une ivoirienne, l'autre burkinabée, puis, à une privatisation de l'ensemble du réseau, entrée en vigueur en 1995 après la création de la Société internationale de transport par rail (SITARAIL). Chacun des deux Etats détient $15 \%$ des parts, la majorité appartenant à des intérêts privés européens. Au Cameroun, la privatisation qui s'est traduite par la 
substitution en 1999 de la Cameroon Railways Company (Camrail) à la Regiefercam mise en place en 1960, s'est accompagnée de la fermeture des petites lignes jugées "non utiles" pour concentrer les efforts d'assainissement sur le Transcamerounais. La gestion du chemin de fer Djibouti-Addis Abeba a été concédée en 2006 à l'opérateur sud-africain Comazar déjà présent dans plusieurs pays africains.

Au Gabon, la privatisation de l'office du chemin de fer Transgabonais (OCTRA) a été un long feuilleton. Finalement, l'affaire s'est traitée entre l'Etat et les deux principaux utilisateurs du chemin de fer : les forestiers, regroupés dans la Société nationale des bois du Gabon (SNBG), et la COMILOG. Après une brève dévolution de la gestion du Transgabonais aux forestiers en 1999-2000, c'est la COMILOG, par l'intermédiaire de la Société d'exploitation du Transgabonais (SETRAG) qui gère, depuis 2003, le chemin de fer dont elle est le principal client. Les diverses formes prises par les privatisations s'adaptent donc aux contextes spécifiques et à la finalité du transport, desserte locale, intégration territoriale, désenclavement, prolongement de l'économie extractive.

\section{Des avantages comparatifs limités au transport minéralier}

En conclusion de ce bref tour d'horizon, il apparaît clairement que la fonction de desserte des territoires qui fut une des raisons d'être de la création de lignes de chemin de fer est aujourd'hui battue en brèche par le transport routier, du moins dans le contexte technologique actuel. Le train ne conserve quelque avantage que pour le transport de marchandises, comme on peut voir par exemple dans les corridors de développement.

Mais l'avenir durable du chemin de fer réside essentiellement dans son rôle irremplaçable pour le transport de produits miniers, y compris en Afrique du sud où les minerais et le charbon représentent plus de $90 \%$ du fret marchandises (Spoornet, 2004). Les grands projets actuels sont tous liés à la croissance de l'économie minière: extension du Transgabonais ; construction d'un Transguinéen destiné à l'exploitation du fer des Monts Nimba au nord de la Guinée; réhabilitation du chemin de fer de Beira au Mozambique, préalable à l'extraction du charbon des mines de Moatize ; réhabilitation du chemin de fer de Benguela pour relancer l'économie katangaise, projet de mise en valeur du fer de la Falémé au Sénégal nécessitant la construction d'un chemin de fer vers un port atlantique, etc. Tous ces projets confirment la place dévolue à l'Afrique dans la mondialisation: fournir des matières premières aux pays du Nord et aux pays émergents. Ils renforcent l'extraversion macro-économique du continent mais leur impact sur le développement local reste limité. Celui-ci dépend désormais de la route, le chemin de fer pouvant au mieux constituer un appoint, en quelque sorte par défaut, là où il n'y a pas de bonne route, par exemple dans la traversée du Mayombe où le Congo-Océan permet aux paysans d'expédier leurs bananes vers Pointe-Noire et Brazzaville. Mais ces situations sont vraisemblablement provisoires, à moins qu'un progrès technologique significatif permette au train de redevenir attractif. Cela supposerait une amélioration considérable du niveau de développement qui ne se profile ni dans le court ni dans le moyen terme.

\section{Conclusion}

$\mathrm{Au}$ train colonial qui joua dans le passé un rôle considérable dans la structuration des territoires, s'est substitué le train de l'économie mondialisée, élargissement de la dissymétrie fondamentale qui régit les relations entre l'Afrique et un Nord désormais 
grossi des pays émergents. Les voies ferrées minéralières ont assurément de l'avenir. Si elles s'intègrent à l'économie-monde, elles ne participent guère à l'intégration territoriale régionale ou nationale et n'ont pas vocation à constituer des réseaux.

L'avenir des autres voies ferrées semble en revanche bien incertain. Les liaisons interurbaines résistent mal à la forte concurrence de la route. Les dessertes urbaines ont peutêtre plus d'avenir, notamment dans les grandes agglomérations de plus en plus asphyxiées par le transport routier, comme par exemple les lignes Dakar-Rufisque, ou Lagos-Ifaw. Le chemin de fer continue pourtant à faire rêver, tout comme au début du $\mathrm{XX}^{\mathrm{e}}$ siècle quand des ingénieurs dessinaient des transsahariens. L'utopie reste d'actualité ainsi qu'en témoignent par exemple les projets ferroviaires présentés par le Niger dans le cadre du NEPAD, ou le projet inter-Etats AFRICARAIL né d'un protocole d'accord signé en 1999 entre le Niger, le Burkina Faso et le Bénin (Bontianti, 2006), avec pour objectif l'interconnexion entre les lignes existantes. Mais tout cela n'a d'autre réalité que les traits tracés sur des cartes et relève plus d'un fantasme géographique que d'une analyse rationnelle des coûts. Le long terme donnera peut-être raison aux utopistes; dans l'immédiat, toutefois, l'horizon du rail africain reste bouché. Au mieux, certaines lignes déjà existantes peuvent être maintenues en l'état ou réhabilitées, mais les investissements en matière de transport au sol vont à la route plutôt qu'aux chemins de fer.

\section{BIBLIOGRAPHIE}

BOSSARD L. (dir.) (2005), Atlas régional des transports et des télécommunications dans la CEDEAO, Abuja, Issy-Les-Moulineaux, CEDEAO - Club du Sahel et de l'Afrique de l'Ouest/OCDE.

ALMEIDA-TOPOR H., CHANSON-JABEUR C., LAKROUM M. (1992), Les transports en Afrique, XIX ${ }^{e}-X X^{e}$ siècles, Paris, L'Harmattan.

BÉRANGER C. (2004), “Mozambique. La ligne de Nacala et le développement durable”, La géographie, Acta géographica, Paris, Société de Géographie.

BONTE P. (2001), La montagne de Fer, la SNIM (Mauritanie) : une entreprise minière saharienne à l'heure de la mondialisation, Paris, Karthala.

BONTIANTI A. (2006), “Un chemin de fer au Niger. Rêve ou réalité ?”, Le Chemin de fer en Afrique, Paris, Karthala-PRODIG-SEDET, pp. 55-68.

CHALÉARD J.L., CHANSON-JABEUR C., BÉRANGER C. (éditeurs scientifiques) (2006), Le chemin de fer en Afrique, Paris, Karthala-PRODIG-SEDET.

CHARLIER J. (1993), La “Voie nationale” du Zaïre, Bruxelles, AESOM.

CHARLIER J., M'PENE Z. (2006), “L'axe ferroviaire Matadi-Kinshasa, clé de voûte du système des transports de la République Démocratique du Congo", Le chemin de fer en Afrique, Paris, KarthalaPRODIG-SEDET, pp. 147-159.

GASCON A. (2006), "Fin du chemin de fer, fin de la Grande Ethiopie. La mort annoncée du chemin de fer de Menelik", Le chemin de fer en Afrique, Paris, Karthala-PRODIG-SEDET, pp. 35-54. 
HUYBRECHTS A. (1970), Transports et structures de développement au Congo. Etude du progrès économique de 1900 à 1970, Paris-La Haye, Mouton.

JADOT O. (1952), "Le programme ferroviaire du Congo Belge”, Bulletin des séances, XXIII, 2, Institut Royal Colonial Belge, pp. 522-544, Bruxelles.

JAEKEL F. (1997), The history of the Nigerian Railway, Ibadan, Nigeria Spectrum Books Limited.

LAKROUM M. (1987), Chemins de fer et réseaux d'affaires en Afrique occidentale : le Dakar-Niger, 1883-1960, Thèse de doctorat d'Etat, Université Paris VII.

LEDERER A. (1982), “Considérations relatives à la Voie nationale du Zaïre”, Bull. de l'Académie royale des sciences d'outre-mer, 28, Bruxelles, pp. 193-208.

MILLER C. (1971), The Lunatic Express, London, Macmillan Publishing Company, réédition 2001, Penguin Classics.

M'PENE Z. (2002), La problématique de l'intégration économique par les transports au Congo, Louvain-laNeuve/Paris, Ed. Academia/L'Harmattan.

MURAIRI J.B. (1970), “Incidences économiques de la jonction des chemins de fer B.C.K. et C.F.M.K. sur la région à traverser”, Kinshasa, Cahiers Economiques et sociaux, VIII, 4, pp. 515-576.

OCDE, BAD (2006), "Perspectives économiques en Afrique”, African Economic Outlook, Paris, Editions de l'OCDE.

POURTIER R. (1982), “Stratégie ferroviaire et politique de l'espace : le Transgabonais”, Hérodote, 25, Paris, pp. 105-128.

POURTIER R. (1993), "Désorganisation des transports et spirale du sous-développement au Zaïre", Politiques agricoles et initiatives locales, Paris, ORSTOM, pp. 49-69.

ROBBINS D. (1993), Blue Train, Johannesburg, Penguin.

SAUTTER G. (1958), Le chemin de fer Bangui-Tchad dans son contexte économique régional. Etude géographique de l'économie des transports au Tchad et dans le nord de l'Oubangui-Chari (une réédition remaniée est parue en 2000 sous le titre Un projet colonial sans lendemain : le chemin de fer BanguiTchad), Paris, EHESS, Centre d'Etudes africaines.

SAUTTER G. (1967), "Notes sur la construction du chemin de fer Congo-Océan (1921-1924)”, Paris, Cahiers d'études africaines, 26, pp. 219-299.

SOUTH AFRICA YEARBOOK 2002-2003 (2003), Pretoria, Government Communication and Information System.

SPOORNET (2004), Annual Divisional Report.

\section{NOTES}

1. Un colloque de géographie consacré aux chemins de fer en Afrique s'est tenu à Paris en septembre 2004. Il a donné lieu à la publication d'un ouvrage collectif, comprenant de nombreuses études de cas (Chaléard et al., 2006).

2. La Haute-Volta, aujourd'hui Burkina Faso, a été reconstituée en 1947. 


\section{RÉSUMÉS}

Les chemins de fer africains ont été les infrastructures de transport les plus emblématiques de la colonisation: outils de contrôle des territoires, d'accès aux espaces enclavés, d'exportation des matières premières. A l'exception de l'Afrique du sud cependant, ils ne constituent pas de véritables réseaux; ils reflètent l'éclatement territorial colonial. Aujourd'hui, les chemins de fer sont confrontés à la concurrence de la route qui condamne de nombreuses lignes. Les avantages comparatifs du rail ne s'affirment que dans le transport des pondéreux, des produits non périssables, comme le montre l'organisation multimodale des transports dans les corridors de développement. Ce sont les voies ferrées minéralières qui offrent les meilleures perspectives dans un contexte de croissance de la production minière. La question de l'avenir du chemin de fer est ainsi posée entre aménagement du territoire et exutoire minier.

African railroads have been the most emblematic transport infrastructures at the time of colonization, allowing control over territories, access to landlocked spaces, export of raw materials. However, if we except South Africa, they reflect the colonial break-up and do not constitute true networks. Today they have to compete with road transport, which spells death for many lines. Comparative advantages of rail are only found in the transport of heavy or non perishable goods, as shown in the multimodal organisation of transports in the development corridors. Ore railroads offer the best prospects in a context of increasing ore production. The future of railroads is therefore at issue between territorial planning and mining outlet.

\section{INDEX}

Mots-clés : chemin de fer, Afrique subsaharienne, corridors de développement, Transgabonais, matières premières, mines, territoire

Keywords : railroad, Subsaharan Africa, development corridors, Transgabonese Railroad, raw materials, mines, territory

\section{AUTEUR}

\section{ROLAND POURTIER}

Professeur, Université Paris 1, Panthéon-Sorbonne, 191, rue Saint-Jacques - 75005 Paris, nrpourtier@wanadoo.fr 